\title{
Spectrofluorometric Probe Method for Examining Preferential Solvation in Binary Solvent Mixtures
}

\author{
WILLIAM E. ACREE, JR., ${ }^{*}$ DENISE C. WILKINS, and SHERYL A. TUCKER \\ Department of Chemistry, University of North Texas, Denton, Texas 76203-5068
}

\begin{abstract}
A spectrofluorometric method is developed to examine preferential solvation of a probe molecule dissolved in binary solvent mixtures. The method assumes that the solvational sphere around every fluorophore is solvated by only one type of solvent component and that each solvated fluorophore contributes to the measured emission intensity. Expressions derived from the model are illustrated with the use of observed fluorescence emission behavior of 3,4-dihydrobenzolghi|perylene dissolved in binary $n$-heptane +1 ,4-dioxane and dibutyl ether + acetonitrile solvent mixtures, which were measured as part of the present study. Index Headings: Fluorescence; Spectroscopic techniques.
\end{abstract}

\section{INTRODUCTION}

Spectroscopic probe techniques provide a convenient experimental means to study preferential solvation. The method involves the use of a probe molecule (such as Reichardt and co-workers' pyridinium- $\mathrm{N}$-phenoxide betaine dye,,$^{1,2} \mathrm{~N}$-alkylpyridinium iodides, ${ }^{3-5}$ pyrene, ${ }^{6,7}$ etc.) that exhibits different spectroscopic characteristics depending upon the properties of the solubilizing media. The probe molecule selectively binds to a specific surface site, partitions into an organized structure, or is preferentially solvated by one of the solvent components. Appearance of new spectral bands, shifts in the absorption and/or fluorescence emission wavelengths, or changes in the emission intensities provide an indication of the micro-environment immediately surrounding the probe.

Preferential solvation arises whenever the proportion of molecules of any given solvent component within the probe's solvational microsphere is not equal to its bulk mole fraction composition, as is depicted in Fig. 1. "True preferential solvation" is extremely difficult, if not impossible, to model rigorously because there is no guarantee that probe-solvent $\mathbf{A}$ and probe-solvent $\mathbf{B}$ molecular interactions remain independent of other solvent molecules within the solvational sphere. Solvent-solvent interactions may lead to synergistic effects. Although not always stated explicitly, most published spectroscopic probe techniques ${ }^{4,5,8-13}$ assume a more idealized situation where solvent-solvent interactions are neglected and the measured spectral response, $R$, in a binary solvent mixture is given by

$$
R=Y_{\mathrm{A}} R_{\mathrm{A}}^{0}+\left(1-Y_{\mathrm{A}}\right) R_{\mathrm{B}}^{0},
$$

a weighted local mole fraction or volume fraction average of the probe's spectral responses in the two pure solvents, $R_{\mathrm{A}}^{0}$ and $R_{\mathrm{B}}^{0}$. Here $Y_{\mathrm{A}}$ and $1-Y_{\mathrm{A}}$ refer to the solvational sphere composition, which may be quite different from

Received 1 April 1993.

* Author to whom correspondence should be sent. the overall bulk liquid-phase composition, $X_{\mathrm{A}}$ and $1-$ $X_{\mathrm{A}}$.

In this short communication, we consider the application of Eq. 1 to polycyclic aromatic hydrocarbon (PAH) solvent polarity probes, which exhibit probe character as evidenced by a systematic variation of fluorescence intensity ratios with solvent polarity. An expression is derived to mathematically describe how the measured emission intensity ratio should vary with binary solvent composition. The newly derived equation is illustrated with the use of the fluorescence behavior of 3,4-dihydrobenzo[ghi]perylene (DHBPe) dissolved in binary $n$-heptane $+1,4$-dioxane and acetonitrile + dibutyl ether solvent mixtures, which was measured as part of the present investigation. 3,4-Dihydrobenzo[ghi]perylene is the only partially hydrogenated PAH molecule found to date to show solvent polarity probe character. Its I $(\sim 380$ $\mathrm{nm}) / \mathrm{II}(\sim 390 \mathrm{~nm})$ emission intensity ratio ranges from a lower value of $\mathrm{DHBPe}=1.12$ in the nonpolar cyclohexane solvent to $\mathrm{DHBPe}=2.10$ in the more polar dimethylsulfoxide. ${ }^{14}$

\section{MATERIALS AND METHODS}

3,4-Dihydrobenzo[ghi]perylene was prepared by hydrogenation of benzo[ghi]perylene with platinum in glacial acetic acid and purified by chromatography. Stock solutions were prepared by dissolving solutes in dichloromethane. Known aliquots of the stock solutions were transferred into test tubes, allowed to evaporate, and diluted quantitatively with the solvent of interest. Final solute concentrations of $10^{-5} \mathbf{M}$ (or less) were sufficiently dilute to minimize inner-filtering artifacts. Solvents were of $\mathrm{HPLC}$, spectroquality, or $\mathrm{AR}$ grade, purchased commercially from Aldrich Chemical Company, and the resulting solutions were optically dilute (absorbance $\mathrm{cm}^{-1}<0.01$ ) at all wavelengths so as to minimize undesired primary and secondary inner-filtering artifacts. ${ }^{15}$ Binary solvent mixtures were prepared volumetrically with burettes so that stoichiometric mole and volume fraction compositions could be calculated to \pm 0.01 or better.

Absorption spectra were recorded on a Bausch and Lomb Spectronic 2000 and a Hewlett-Packard 8450A photodiode array spectrophotometer in the usual manner. The fluorescence spectra were run on a Shimadzu $\mathrm{RF}-5000 \mathrm{U}$ spectrofluorometer with the detector set at high sensitivity. Solutions were excited at $334 \mathrm{~nm}$. Fluorescence data were accumulated in a quartz $1-\mathrm{cm}^{2} \mathrm{cu}-$ vette at $19^{\circ} \mathrm{C}$, ambient room temperature, with excitation and emission slit width settings of $15 \mathrm{~nm}$ and $3 \mathrm{~nm}$, 
respectively. Emission spectra obtained represent a single scan which was then solvent blank corrected and verified by repetitive measurements.

\section{RESULTS AND DISCUSSION}

From a theoretical standpoint, variation of emission intensity ratios with solvent composition can be mathematically modeled in a relatively straightforward manner. The solvational sphere around every PAH fluorophore is assumed to be solvated by only one type of solvent molecule, either by solvent $\mathbf{A}$ or by solvent $\mathbf{B}$, as depicted in the simulated preferential solvation scheme of Fig. 1. Extent of preferential solvation is thus determined by the relative mole numbers of each solvational sphere type, rather than by the local mole fraction composition within the solvation sphere. Each solvated fluorophore contributes to the observed fluorescence signal, $F_{\text {obs }}$, at each emission wavelength scanned:

$$
F_{\text {obs }}=K_{\text {fluoro A }}^{\prime}\left(P_{0}-P_{\text {fluoro A }}\right)+K_{\text {fluoro } \mathbf{B}}^{\prime}\left(P_{0}-P_{\text {fluoro B }}\right)
$$

where $P_{0}$ refers to the intensity of the incoming monochromatic excitation radiation and $\left(P_{0}-P_{\text {fluoro } i}\right)$ is the amount of radiation absorbed by solvated fluorophore type $i$. The two proportionality constants, $K_{\text {fuoro }}^{\prime}$ and $K_{\text {fluoro }}^{\prime}$, depend upon the various optical component placements within the instrument, detector response/ efficiency, and quantum yield of the given solvated fluorophore.

The Beer-Lambert law relates the intensity of unabsorbed excitation radiation, $P_{\text {fluoro } i}$, to

$$
P_{\text {fluoro } i}=P_{0} 10^{-b \epsilon_{\text {fluors } i} i \text { Fluoro } i \text { ) }}
$$

molar concentration of fluorophore, [Fluoro $i$ ], and the molar extinction coefficient, $\epsilon_{\text {fluoro } i}$. Substitution of Eq. 3 into Eq. 2 gives

$$
\begin{aligned}
F_{\text {obs }}= & K_{\text {fluoro A }}^{\prime} P_{0}\left(1-10^{-b_{\text {fluoro A }}[\text { Fluoro A] }}\right) \\
& +K_{\text {fluoro B }}^{\prime} P_{0}\left(1-10^{\left.-b \epsilon_{\text {flurro }} \text { B [Fluoro B }\right]}\right)
\end{aligned}
$$

which can be expanded as a Maclaurin power series to yield

$$
\begin{aligned}
& F_{\text {obs }}=K_{\text {fluoro A }}^{\prime} P_{0}\left\{2.303 b \epsilon_{\text {fluoro } \mathrm{A}}\right. \text { [Fluoro A] } \\
& -\left(2.303 b \epsilon_{\text {fluoro A }} \text { [Fluoro A ] }\right)^{2} / 2 \text { ! } \\
& +\left(2.303 b \epsilon_{\text {fluoro }}\left[\begin{array}{l}
\text { Fluoro A } \\
\mathbf{A}
\end{array}\right)^{3} / 3 !-\ldots\right\} \\
& +K_{\text {fluoro B }}^{\prime} P_{0}\left\{2.303 b \epsilon_{\text {fluoro }}\right. \text { [Fluoro B] } \\
& -\left(2.303 b \epsilon_{\text {fluoro }} \text { [ [Fluoro B] }\right)^{2 / 2} \text { ! } \\
& \left.+\left(2.303 b \epsilon_{\text {fluoro } \mathbf{B}}[\text { Fluoro } \mathbf{B}]\right)^{3} / 3 !-\ldots\right\} \text {. }
\end{aligned}
$$

For very dilute solutions where $2.303 b \epsilon_{\text {fluoro } i}[$ Fluoro $i]<$ 0.05 , the higher-order terms are negligible. With this simplification, the measured emission is

$$
\begin{aligned}
F_{\text {obs }}= & 2.303 K^{\prime}{ }_{\text {fluoro } \mathbf{A}} P_{0} b \epsilon_{\text {fluoro }} Y_{\mathbf{A}} \text { [Fluoro] } \\
& +2.303 K_{\text {fluoro } \mathbf{B}} P_{0} b \epsilon_{\text {fluoro } \mathbf{B}}\left(1-Y_{\mathbf{A}}\right) \text { [Fluoro] }
\end{aligned}
$$

whenever expressed in terms of the total stoichiometric fluorophore concentration, [Fluoro]. Here, $Y_{\mathrm{A}}$ and 1 $Y_{\mathrm{A}}$ represent the mole number fraction of each type of solvated fluorophore, i.e., $Y_{\mathbf{A}}=[$ Fluoro $\mathrm{A}] /[$ Fluoro $]$ and $1-Y_{\mathbf{A}}=$ [Fluoro B]/[Fluoro]. Inherent in the above treatment is the underlying assumption that neither sol-

\section{TRUE PREFERENTIAL SOLVATION}

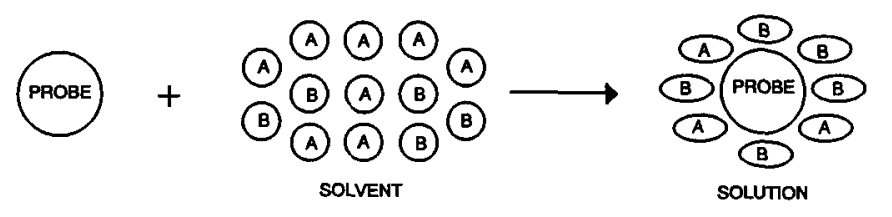

SIMULATED PREFERENTIAL SOLVATION

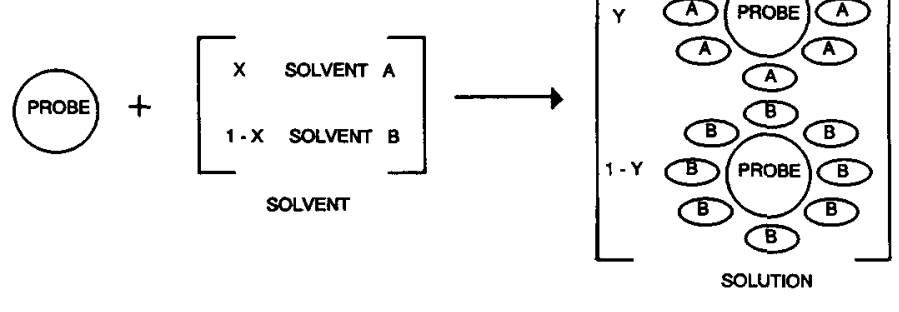

FIG. 1. Schematic representation depicting important differences between true preferential solvation and models commonly used to simulate preferential solvation in binary solvent mixtures. In the simulated case, the solvational sphere around every probe molecule is assumed to be solvated by only one type of solvent component. Extent of preferential solvation is thus determined by the relative mole numbers of each solvational sphere type, rather than by the local mole fraction composition within the solvation sphere, as is the actual case.

vent component forms a nonfluorescent association complex with the fluorophore. If such complexation does occur, then Eq. 6 describes only the fraction of the solute molecules that actually fluoresce.

Examination of Eq. 6 reveals that the observed PAH emission spectra for a binary solvent mixture is a weighted mole fraction average of the fluorophore's spectra in each of the two pure solvents, provided that the molar concentration of fluorophore remains constant for each series of measurements. Emission intensities are additive at each wavelength. In the case of a PAH solvent polarity probe such as 3,4-dihydrobenzo[ghi]perylene, the calculated I/II emission intensity ratio is

$$
\begin{aligned}
\mathrm{I} / \mathrm{II} \approx & {\left[Y_{\mathbf{A}} \mathrm{I}_{\text {solvent } \mathbf{A}}+\left(1-Y_{\mathbf{A}}\right) \mathrm{I}_{\text {solvent } \mathbf{B}}\right] } \\
& \div\left[Y_{\mathbf{A}} \mathrm{II}_{\text {solvent A }}+\left(1-Y_{\mathbf{A}}\right) \mathrm{II}_{\text {solvent } \mathbf{B}}\right],
\end{aligned}
$$

also determined by the extent of preferential solvation. Here, we have assumed that both the I and II band emission wavelengths are solvent independent, which is not strictly true; hence the "approximately equal to" sign is used. Rigorous applications require that intensity measurements be made at two fixed emission wavelengths. It is only under the very special set of circumstances where $\mathrm{II}_{\text {solvent } \mathrm{A}}=\mathrm{II}_{\text {solvent } \mathrm{B}}$ that the emission intensity ratio equals

$$
\mathrm{I} / \mathrm{II}=Y_{\mathrm{A}}(\mathrm{I} / \mathrm{II})_{\text {solvent A }}+\left(1-Y_{\mathrm{A}}\right)(\mathrm{I} / \mathrm{II})_{\text {solvent } \mathbf{B}},
$$

a simple weighted fraction average of ratios in the pure solvents. Nakashima et al. ${ }^{16}$ employed Eq. 8 expressed in terms of I/III to investigate pyrene partitioning among polystyrene (PS) and poly(2-vinylpyridine) (PVP) microdomains in PS-PVP diblock copolymers. To our knowledge, there is no experimental evidence to justify the authors' assumption.

Application of Eq. 7 is relatively straightforward if the spectrofluorometer is equipped with data processing and 


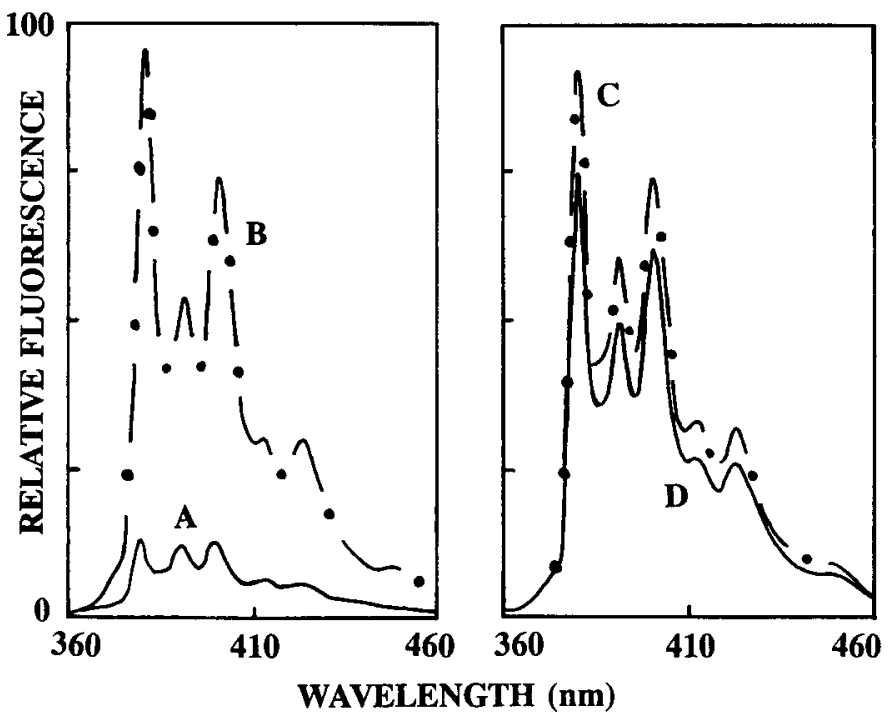

Fig. 2. Fluorescence emission behavior of 3,4-dibenzo[ghi]perylene dissolved in neat $n$-heptane $[(A)(-)]$, in neat 1,4-dioxane $[(B)$ $(--)]$, and in a binary $n$-heptane-dioxane mixture $[(C)(-\bullet-)]$ having a stoichiometric volume fraction composition of $\phi_{\text {heptane }}=0.50$. Spectrum $D(-)$ represents the emission spectrum calculated with the use of Eqs. 6 and 7, with a preferential solvation mole fraction of $Y_{\text {heptanc }}=0.74$. After normalization to a common band intensity, spectra $C$ and $D$ are superimposable.

manipulation software. The fluorescence emission spectra are recorded for the PAH probe dissolved in both pure solvents, and the measured I and II band intensities are substituted into the numerator and denominator, respectively. "Trial" values of $Y_{\mathrm{A}}$ and $1-Y_{\mathrm{A}}$ are computed from the measured I/II ratio for each binary solvent composition studied. These trial values are then used in Eq. 6 to generate the calculated fluorescence emission spectra, which are compared to the observed data. Careful attention is given to ensure that the entire detailed emission fine structure (wavelengths and all intensity ratios) is correctly produced, rather than just the experimental I/II ratio. If necessary, the trial values can be adjusted repetitively until the "best possible" agreement between observed and calculated spectra is achieved, as shown in Fig. 2. Spectra $A$ and $B$ in this figure represent the emission intensities of 3,4-dihydrobenzo[ghi]perylene dissolved in neat $n$-heptane and 1,4dioxane, respectively, scanned from $360-460 \mathrm{~nm}$. A trial value of $Y_{\text {heptane }}=0.74$ reproduced very accurately the observed dihydrobenzo[ghi]perylene emission spectra at the binary solvent volume fraction composition of $\phi_{\text {heptane }}$ $=0.50$ after normalization to a common band intensity (spectra $C$ vs. spectra $D$ in Fig. 2). Normalization corrects for small differences in experimental conditions, such as fluorophore concentration, ambient room temperature, and excitation source intensity, which may occur during any given series of fluorescence measurements. In most cases there was less than a $1 \%$ difference between the observed and calculated spectra after normalization.

Figure 3 summarizes results of our preferential solvation computations for 3,4-dihydrobenzo[ghi]perylene dissolved in binary $n$-heptane $+1,4$-dioxane and dibutyl ether + acetonitrile mixtures. The nine stoichiometric binary compositions are represented as both mole (e)

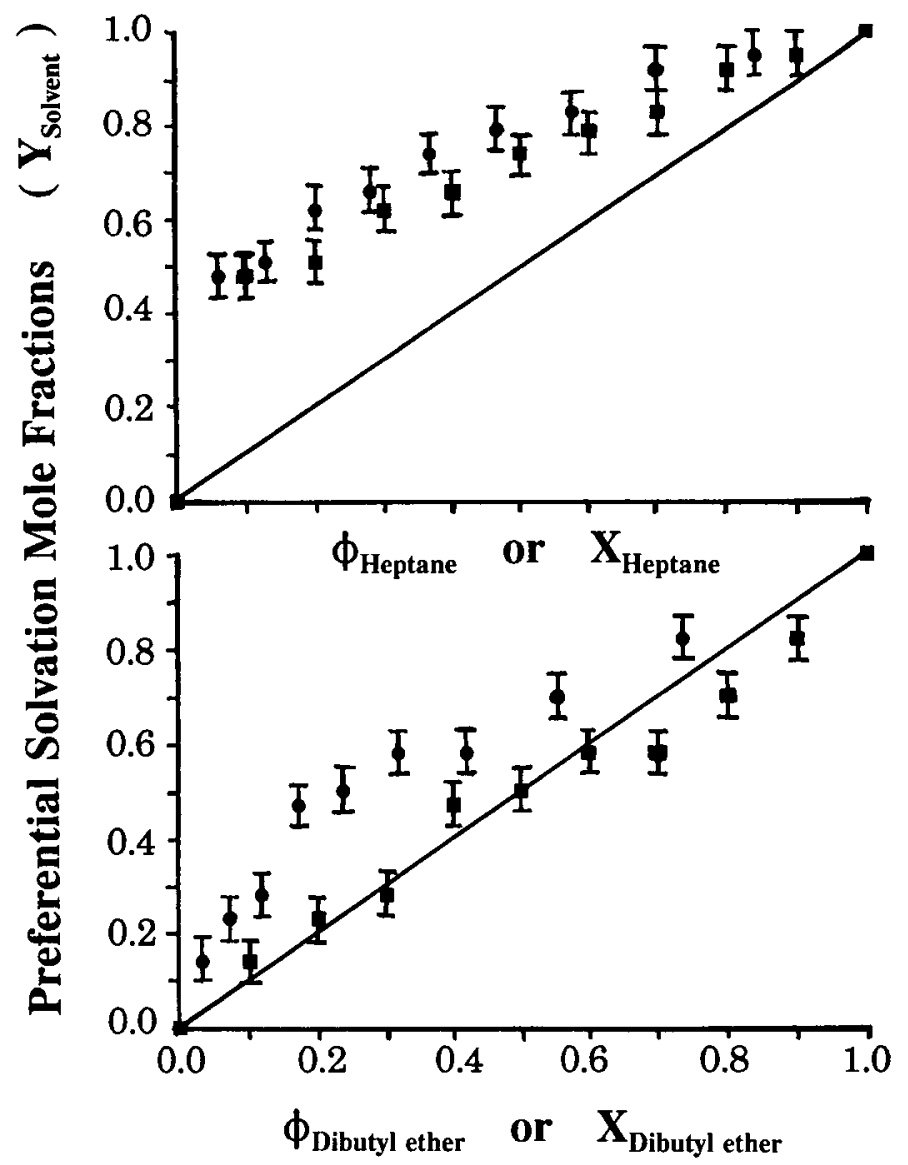

FIG. 3. Preferential solvation of 3,4-dihydrobenzo[ghi]perylene dissolved in $n$-heptane $+1,4$-dioxane (upper set of curves) and dibutyl ether + acetonitrile (lower set of curves) mixtures. Numerical values of $Y_{\text {heptane }}$ and $Y_{\text {dihutyl ether }}$ were calculated from Eq. 7 with the use of observed I (at $\sim 380) /$ II ( at $\sim 390$ ) emission intensity ratios. The $\mathbf{x}$-axis denotes the stoichiometric mole $(\bullet)$ and volume (ठ) fraction compositions of the binary solvent.

and volume ( $\square$ ) fractions. Uncertainties assigned to the various $Y_{\text {heptane }}$ and $Y_{\text {dibutyl ether }}$ values, indicated by the error bars, were based upon the reproducibility of the emission intensity ratios (circa \pm 0.02 or so) and the range of possible "trial" values that predicted identical I/II ratios. For example, if numerical values of $\mathrm{I}_{\text {heptane }}=48.3$, $\mathrm{I}_{\text {dioxane }}=365.2, \mathrm{II}_{\text {heptane }}=43.2$, and $\mathrm{II}_{\text {dioxane }}=207.6$ for 3,4 dihydrobenzo[ghi]perylene are substituted into Eq. 6, then weighted fractions ranging from $Y_{\text {heptane }}=0.17$ to $Y_{\text {heptane }}=0.21$ give exactly the same intensity ratio of I/II $=1.73$ when rounded to the second decimal place.

Examination of Fig. 3 reveals that the computational method returned fairly smooth curves for how the degree of presumed preferential solvation varies with solvent composition. In the ideal case, where the solvational sphere micro-environment is governed exclusively by the relative mole numbers of both solvent components, the local composition around the solute probe should equal the solution's stoichiometric mole fraction. For binary $n$-heptane +1 ,4-dioxane mixtures there is an unexpectedly large local composition of the nonpolar $n$-heptane cosolvent around the polycyclic aromatic hydrocarbon probes. Naively, one would have expected that dipoleinduced dipole interactions involving the oxygen lone electron pairs on the ether and the PAH's polarizable 
$\pi$-electron cloud would result in preferential solvation that would favor the more polar 1,4-dioxane cosolvent. Such interactions, however, may have led to the formation of nonfluorescent PAH-dioxane association complexes, and would not have been properly accounted for in this particular preferential solvation scheme. As noted previously, Eq. 7 assumes that each solvated fluorophore contributes to the observed fluorescence signal. Alternatively, the unexpected preferential solvation of $n$-heptane around the PAH fluorophore may be a calculational artifact resulting from the large differences in emission intensities observed for 3,4-dihydrobenzo[ghi]perylene dissolved in pure $n$-heptane vs. that in pure 1,4-dioxane. Mathematically, one must use a relatively large fraction of the DHBPe spectra in $n$-heptane to reproduce the emission intensity ratios observed in binary $n$-heptane + 1,4-dioxane mixtures. Unfortunately, this happens to be one of the limitations of this particular spectrofluorometric probe method which is based upon intensity ratios of select emission bonds.

In closing, readers are reminded that it is fundamentally impossible to prove that a particular spectroscopic probe method is correct. One can demonstrate, however, that a given method is consistent with a wide range of experimental observations, which implies that the method and assumptions made therein may be correct. Similarly, it can be shown that a given spectroscopic probe method is inconsistent with experimental data so that the method must be either incorrect or incomplete. It is hoped that the ideas presented in this paper will prompt a critical re-examination of the relative merits of the various spectrofluorometric probe methods, Eq. 7 vs. Eq. 8 , in hopes of achieving a better understanding of solutesolvent interactions in fluid solution.

\section{ACKNOWLEDGMENTS}

This work is supported in part by grants from the National Science Foundation (Grant No. CTS-8922485), by the University of North Texas Research Council, and by a National Science Foundation Doctoral Research Fellowship awarded to S. A. Tucker. The authors thank J. C. Fetzer for kindly providing the sample of 3,4-dihydrobenzo[ghi]perylene.

1. K. Dimroth, C. Reichardt, T. Siepmann, and F. Bohlmann, Liebigs Ann. Chem. 661, 1 (1963).

2. C. Reichardt, Solvents and Solvent Effects in Organic Chemistry (VCH Publishers, New York, NY, 1988), 2nd ed.

3. P. Chatterjee, A. K. Laha, and S. Bagchi, J. Chem. Soc., Faraday Trans. 88, 1675 (1992).

4. P. Chatterjee and S. Bagchi, J. Phys. Chem. 95, 3311 (1991).

5. P. Chatterjee and S. Bagchi, J. Chem. Soc., Faraday Trans. 86, $1785(1990)$.

6. D. C. Dong and M. A. Winnik, Can. J. Chem. 62, 2560 (1984).

7. K. Kalyanasundaram and J. K. Thomas, J. Am. Chem. Soc. 99, 2039 (1977).

8. W. P. Zurawsky and S. F. Scarlata, J. Phys. Chem. 96, 6012 (1992).

9. E. Bosch and M. Roses, J. Chem. Soc., Faraday Trans. 88, 3541 (1992).

10. P. Chatterjee and S. Bagchi, J. Chem. Soc., Faraday Trans. 87, 587 (1991).

11. K. Tamori, Y. Watanabe, and K. Esumi, Langmuir 8, 2344 (1992).

12. Y. P. Sun, G. Bennett, K. P. Johnston, and M. A. Fox, J. Phys. Chem. 96, 10001 (1992).

13. M. Szpakowska and O. B. Nagy, J. Chem. Soc., Faraday Trans. I 85, 2891 (1989).

14. S. A. Tucker, W. E. Acree, Jr., J. C. Fetzer, and R. H. Mitchell, Appl. Spectrosc. 47, 1040 (1993).

15. K. W. Street, Jr., and W. E. Acree, Jr., Analyst 111, 1197 (1986).

16. K. Nakashima, M. A. Winnik, K. H. Dai, E. J. Kramer, and J. Washiyama, Macromolecules 25, 6866 (1992). 